\title{
Dynamic Spectrum Sharing Optimization and Post-optimization Analysis with Multiple Operators in Cellular Networks
}

\author{
Md Asaduzzaman, Raouf Abozariba* and Mohammad N. Patwary \\ $\{$ md.asaduzzaman $\},\{$ r.abozariba $\},\{$ m.n.patwary\}@staffs.ac.uk \\ School of Creative Arts and Engineering \\ Staffordshire University, Stoke-on-Trent, Staffordshire ST4 2DE, United Kingdom
}

\begin{abstract}
Dynamic spectrum sharing aims to provide secondary access to under-utilised spectrum in cellular networks. The main aim of the paper is twofold. Firstly, secondary operator aims to borrow spectrum bandwidths under the assumption that more spectrum resources exist considering a merchant mode. Two optimization models are proposed using stochastic and optimization models in which the secondary operator (i) spends the minimal cost to achieve the target grade of service assuming unrestricted budget or (ii) gains the maximal profit to achieve the target grade of service assuming restricted budget. Results obtained from each model are then compared with results derived from algorithms in which spectrum borrowings are random. Comparisons showed that the gain in the results obtained from our proposed stochastic-optimization framework is significantly higher than heuristic counterparts. Secondly, post-optimization performance analysis of the operators in the form of blocking probability in various scenarios is investigated to determine the probable performance gain and degradation of the secondary and primary operators respectively. We mathematically model the sharing agreement scenario and derive the closed form solution of blocking probabilities for each operator. Results show how the secondary operator perform in terms of blocking probability under various offered loads and sharing capacity.
\end{abstract}

Keywords: Dynamic spectrum sharing, spectrum allocation, merchant mode, spectrum pricing, aggregated channel allocation algorithms.

\footnotetext{
*Corresponding author: Raouf Abozariba, Staffordshire University, College Road, Stoke-on-Trent, Staffordshire ST4 2DE, United Kingdom. Mob: +447429234875, Email: r.abozariba@staffs.ac.uk.
} 


\section{INTRODUCTION}

\section{A. Background and motivation}

The static partitioning of spectrum in cellular networks has significant operational implications, (e.g., pseudo scarcity of the available radio spectrum) which have been identified by extensive spectrum utilisation measurements $[1,2]$. These measurements show that a large part of the spectrum, which is allocated to cellular use, are well utilised, but the utilisation varies dramatically over time and space. Such variation of spectrum utilisation causes the so-called spectrum holes, which can be opportunistically utilised to improve the network's grade of service (GoS) $[3,4]$. The grade of service is generally defined by the level of blocking probability, where higher blocking probability means lower grade of service [5]. Depending on type of bandwidth $(800 \mathrm{MHz}, 1.8 \mathrm{GHz}, 2.5 \mathrm{GHz})$ in a cell, location of the cell, number of users, demand of spectrum may vary significantly and GoS is often degraded. Therefore, operators would require additional spectrum in high demand periods to improve their GoS. A solution to increase the efficiency of spectrum utilization by means of sharing has been addressed in the research domain [68]. Spectrum sharing between operators often results in a considerable improvement of GoS, although it would incur additional costs to the operators [9-12]. Since network operators often operate with a limited budget, the borrowing decisions of a network operator could be affected. Consequently, the operators would need to make dynamic, on-demand and correct choices of borrowing additional bandwidths from other operators [13].

Given a market scenario with several primary and secondary operators, where secondary network operators (SNOs) are operators which obtain spectrum resources via dynamic spectrum leasing from primary network operators (PNOs) which are the incumbent holders of spectrum licenses, and also rules and conditions of spectrum access, spectrum requirement and their prices, and other parameters, our main idea is to optimize the resource sharing among such operators under a target GoS and budget restriction for the SNOs. We propose two algorithms: the first is to optimize the amount of savings that SNOs could achieve when they engage in spectrum trading with PNOs to gain a certain threshold of GoS. The second is to optimize the profit of SNOs under budget restrictions.

The targeted GoS can not be always guaranteed due to the mutual spectrum sharing agreement between the operators. Therefore, a post-optimization analysis is needed to calculate the actual 
GoS in terms of blocking probability after borrowing resources from the PNOs. Hence, we derive the blocking probability formulae under a mutual agreement to share spectrum where the leased spectrum bandwidth can be deviated according to the operators internal demand. We allow operators to dynamically access or handover part of the shared spectrum according to their internal demand state. Major contributions of this paper are summarized as follows:

- a novel purchase approach for dynamic spectrum sharing (DSS) network is proposed in the presence of multiple secondary and primary network operators. We introduce two optimization problems in merchant mode DSS,

- the robustness of the proposed algorithms are investigated in the presence of large number of cells and various types of spectrum bandwidths and the proposed algorithms are compared with heuristic borrowing algorithm. Comparisons show a substantial gain over the heuristic borrowing algorithms and

- a post-optimization analysis technique of the operators' performance (secondary and primary) in the form of blocking probability is derived, which gives the actual GoS of the operators after resource sharing.

The dynamic spectrum management framework discussed in this paper can support some core functions of various systems such as the automation of licensing and Spectrum Access System (SAS), which perform a number of functions such as real-time assessment of spectrum availability, determine the available frequencies at a given geographic location, interference protection, operational privacy, enforcement of regulatory policies, and coexistence techniques [14].

\section{B. Related work}

In the literature, a great number of studies has appeared in recent years on the design of dynamic spectrum sharing within cellular networks [10,15-19]. Interests in this context include secondary leasing and pricing strategies among incumbent spectrum license holders, SNOs and secondary users. These prior studies mainly focused on approaches using auction mode and game theory to implement the spectrum pricing and allocation schemes by taking into account the variation of the networks demands and constraints such as power, price and interference [10, $11,15-18,20]$. 
In [20], the authors proposes a multiple-dimension auctioning mechanism through a broker to facilitate an efficient secondary spectrum market. Auction schemes where a central clearing authority auctions spectrum to bidders, while explicitly accounting for communication constraints is proposed in [21]. While in [22], spectrum auctions in a dynamic setting where secondary users can change their valuations based on their experiences with the channel quality was studied. Price-based DSS has also been investigated from the business perspective [23, 24]. For example, in [25] An extensive business portfolio for heterogeneous networks is presented to analyse the benefits due to multi-operator cooperation for spectrum sharing. High resolution pricing models are developed to dynamically facilitate price adaptation to the system State. In [26], a qualityaware dynamic pricing algorithm (QADP) which maximizes the overall network revenue while maintaining the stability of the network was studied.

The vast majority of the aforementioned studies consider competitive market scenarios and therefore auction and game theory have been discussed to develop DSS strategies. By using the same assumption, pricing in the context of DSS has mainly been considered from the spectrum owners perspective to maximize their revenues $[18,23,27]$. However, when the number of available bandwidths from multiple license owners is higher than SNOs' demand, then auction mode is not always the best strategy. This is because the number of bidders might be too small and the best selling price can not be achieved for the license owners by using auction mode. A more realistic and pragmatic model in this case is a merchant mode, which to the best of our knowledge, has not been investigated in the context of DSS. Moreover, spectrum borrowing when considering budget restrictions has not been addressed. Also, there is currently no published work, which attempted to study the admission cost minimization in the merchant mode with target performance. Thus the problems that we formulate and solve substantially differ from those available in the literature.

The analysis of blocking probability and dynamic aggregated channel assignment has been extensively considered in the context of cellular networks [28, 29]. However, there are significant differences between auction mode and the focus of our work. For example, in auction mode network operators are not assumed to claim back the leased spectrum within a single trading window during busy intervals [30]; whereas in our approach, the leased capacity is dynamic in size. To the best of our knowledge, the proposed post-optimization analysis in this paper is the 
first to study the blocking probability during a trading window with the presence of multiple operators. It also addresses the issue of PNOs' change in state during a single trading window.

The paper is organised as follows: the proposed dynamic spectrum management model is described in Section II. Section III addresses the problem of spectrum allocation in cellular networks and describes our mathematical programming formulations to the problem. Section III-G, presents blocking probability analysis under resource sharing with multiple PNOs. In Section IV, we present our findings. Finally, Section V summarises our conclusions.

\section{DYNAMIC SPECTRUM MANAGEMENT MODEL}

We consider a cellular network to consists of $S$ secondary network operators (SNOs) and $\mathcal{N}$, with size $|\mathcal{N}|=N$, denote the set of primary network operators (PNOs) serving a region $\mathcal{R}$, see Figure 1. Let $\mathcal{L}$, with size $|\mathcal{L}|=L$, be the set of cells in the region.

Each primary and secondary operator in the network is licensed with an incumbent bandwidth consisting of a set of component carriers, each of which can be allocated to support the operators' subscribers. The antenna towers/masts at the centre of each cell $i \in \mathcal{L}$ is shared among the operators. The operators are managed by radio network controllers (RNC) which monitor base stations and assign resources for the air interface as well as other functions $[31,32]$. In the context of this cellular networks arrangement, we only consider cells with an almost identical radio environment, which is visible to all providers in each cell. An example of this setup is when a town or city requires operators to use common towers for their antennas, due to economy of scale property of telecommunication industry.

Due to spectrum liberalisation, the PNOs $|\mathcal{N}|$ will have the freedom to lease their spectrum bandwidths to the SNOs. Leasing spectrum bandwidths would mean that the SNO will have to pay a certain compensation to the PNO for using the spectrum bandwidths, and the amount of compensation is expected to be proportional to the amount of allowed spectrum leasing by the primary system. We assume the compensation paid to the PNO is in form of monetary value. The PNOs broadcast specific information about their available bands for leasing and admission cost (per unit bandwidth) at each cell $i \in \mathcal{L}$ at fixed identical intervals (e.g., every 2 hours). The lease conditions may specify additional parameters such as the extent of spatial region for spectrum use and maximum power. The compliant use of leased spectrum requires that the SNOs return 
the spectrum to the PNO at the end of the lease interval. The duration of each lease could be decided by the network providers under a mutual agreement, and/or any other regulatory bodies' conditions (e.g., minutes, hours, days). In our model, we assume that the PNOs receive a series of demands from multiple SNOs at different time instances [25,33]. Depending on the time of SNO's request, different sets of frequencies and prices can be available. Demands are awarded instantaneously, in which case the borrowed spectrum can be immediately used. We consider the spectrum sharing problem among multiple SNOs, which support different types of spectrum bands and hence have heterogeneous GoS demands at different cells.

The available channels may vary in the bandwidth and transmitting range to support different sizes of cells and cells with different terrain characteristics. The connectivity and scalability of the network varies because a channel with shorter transmission range may not cover all the areas covered by a channel with a longer transmission range. Thus, there is an obvious need for further band's categorisation prior to spectrum trading, which takes heterogeneity of cells into account. We address this issue by considering multiple types of spectrum bands, assuming that each have different characteristics (e.g., $800 \mathrm{MHz}, 1.8 \mathrm{GHz}$ and $2.5 \mathrm{GHz}$ bands). This will be useful when considering $5 \mathrm{G}$ networks where a variety of bands are expected to be in deployment.

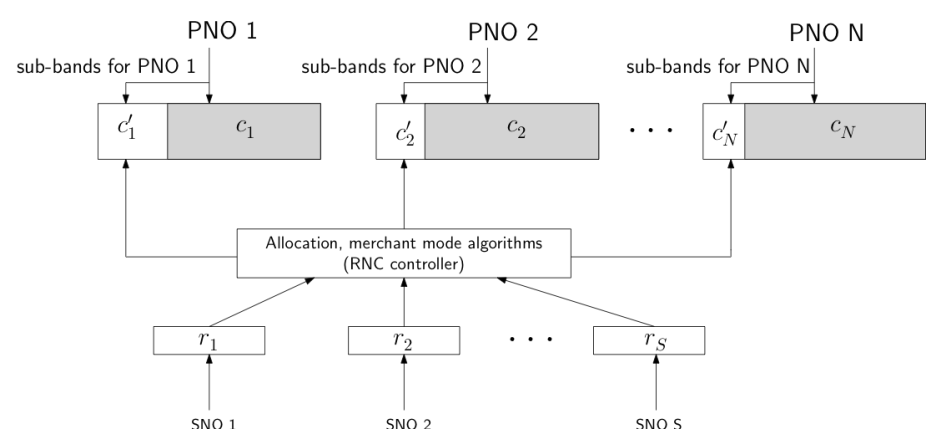

Fig. 1: Network model for cellular network with $N$ PNOs and $S$ SNOs

\section{PROBLEM FORMULATION}

Considering the system model described in Section II, the problem now becomes how the RNC of an SNO acquires additional spectrum from the PNOs. The spectrum borrowing can be performed by considering one of the following objectives:

- to minimize borrowing cost in each time slot by selecting the lowest cost combinations of available spectrum from the primary networks to achieve a specified GoS and 
- to maximize profit in each time slot by borrowing the highest profit combinations of available spectrum from the primary networks under restricted budget to achieve a specified GoS.

In principle, the RNC's objective is to minimize overall operating cost or to maximize revenue for an SNO as well as to maximize utility to the end users.

\section{A. Modelling assumptions}

In this subsection we identify the part of network information which is assumed to be known to the SNOs:

- arrival rate of the $s$ th $\mathrm{SNO}$ at $i$ th cell for $j$ th type of spectrum band $\lambda_{s i_{j}}, \forall s, i, j$,

- service rate of the $s$ th SNO at $i$ th cell for $j$ th type of spectrum band $\mu_{s i_{j}}, \forall s, i, j$,

- available bandwidth of the $s$ th SNO at $i$ th cell for $j$ th type of spectrum band $w_{s i}, \forall s, i, j$,

- borrowing cost of the sth SNO for unit bandwidth from the PNOs at $i$ th cell for $j$ th type of spectrum band $c_{i_{j} k}, \forall i, j, k$ (which are assumed to be announced periodically by the PNOs),

- allocated budget for borrowing bandwidths to the $s$ th SNO at $i$ th cell for $j$ th type of spectrum bandfrom the PNOs $b_{s i}, \forall s, i, j$,

- available bandwidth of the $k$ th PNO at $i$ th cell for $j$ th type of spectrum band $a_{i_{j} k}, \forall i, j, k$, (which are assumed to be announced periodically by the PNOs), and

Time is divided into equal-length slots $\mathcal{T}=\{0,1,2, \ldots\}$. At each time slot $t \in \mathcal{T}$ the process of aggregated channel borrowing is repeated. We use the time indicator $(t)$ to emphasise the vectors dependancy in time. Trading of bandwidth is done between primary and secondary providers separately in each of successive time windows of a particular duration. Henceforth, we focus on the the process of channel borrowing and optimization in a single window.

\section{B. Notations used in Problem 1 and Problem 2}

Let us define the following quantities which are used later in mathematical programming problems (Problem 1 and Problem 2):

$c_{i_{j} k}(t):=$ cost of unit bandwidth to be borrowed from $k$ th PNO for $j$ type resource at $i$ th cell

during time interval $t$, where $c_{i_{j} k}(t) \in \mathbb{R}_{\geq 0}^{L \times N_{i_{j}}}\left(c_{i_{j} k}(t)>0\right)$ and $N_{i_{j}}$ is the number of PNOs in the $i$ th cell for $j$ th type of service and $L$ is the number of cells in the region $\mathcal{R}$. 
$x_{i_{j} k}(t):=$ unit of spectrum bandwidths (or sub-bands) to be borrowed from $k$ th PNO for $j$ type resource at $i$ th cell during time interval $t$, where $x_{i_{j} k}(t) \in \mathbb{R}_{\geq 0}^{L \times N_{i_{j}}}$.

$\theta_{i_{j} k}(t):=$ PNOs intrinsic quality (e.g., the extent of the coverage area and/or maximum allowable transmit power), where $\left\{\theta_{i_{j} 1}, \theta_{i_{j} 2}, \ldots, \theta_{i_{j} k}, \ldots, \theta_{L \times N}\right\}$.

$p_{s i_{j}}(t):=$ target blocking probability for $j$ type resource at $i$ th cell during time interval $t$ for the secondary network operator.

$a_{i_{j} k}(t):=$ unit bandwidth available from $k$ th PNO to be leased to $s$ th SNO for $j$ th type resource at the $i$ th cell during time interval $t$, where $a_{i_{j} k}(t) \in \mathbb{R}_{\geq 0}^{L \times N_{i_{j}}}$.

$r_{s i_{j}}(t):=$ unit bandwidth required to satisfy the target blocking probability $p_{i_{j}}(t)$ for the $s$ th SNO's for $j$ th type resource at $i$ th cell during time interval $t$, where $r_{i_{j}}(t) \in \mathbb{R}_{\geq 0}^{L}$.

$\gamma_{i_{j} k}(t):=$ the expected profit for borrowing unit bandwidth from $k$ th PNO for $j$ th type resource at $i$ th cell during time interval $t$, where $\gamma_{i_{j} k}(t) \in \mathbb{R}^{L \times N_{i_{j}}}$.

\section{Spectrum allocation by minimising borrowing cost}

We now formulate the spectrum allocation problem, that is, how much spectrum bandwidths to be borrowed from each PNO to keep the blocking probability in a specific level, for instance, at $1 \%$. Given a set of possible available spectrum resources $\left\{a_{i_{j} k}(t)\right\}$ and their associated prices $\left\{c_{i_{j} k}(t)\right\}$, the problem is to find the feasible set of spectrum bandwidths $\left\{x_{i_{j} k}(t)\right\}$ by minimising the total borrowing cost. The PNOs set their prices according to the maximum allowed transmit power $\varpi_{i_{j} k}$ and the pricing coefficient $\varphi_{i_{j} k}$, which can be expressed as [9]

$$
c_{i_{j} k}=\left(\sum_{k \in a_{i_{j} k}}\left[\log \left(1+\frac{h \varpi_{i_{j} k}}{\varrho_{i}}\right)-\left(\varpi_{i_{j} k} \cdot \varphi_{i_{j} k}\right)\right]\right) \cdot\left(a_{i_{j} k}\right)^{-1}
$$

where $h$ is the average aggregated channel gain, $\varrho_{i}$ is the additive noise received by SNO users at cell $i$ and $\varphi_{i_{j} k}$ represents pricing coefficient of PNO $k$ for the SNO in the $i$ th cell for causing each unit of interference. Equation (1) shows that PNOs select prices in a way such that the collective preference order of transmit power, channel gain and noise are retained. This cancels the intuition that prices are selected so that all channels available for borrowing are equally preferable to a secondary. In addition, each PNO incurs a minimum cost $X_{(\mathrm{min})}$ when it leases 
its channel to the SNOs and therefore it is not possible to select a price lower than $X_{(\min )}$ such that

$$
c_{i_{j} k}= \begin{cases}\text { RHS of Eq. (1), } & \text { RHS of Eq. (1) } \geq X_{(\min )} \\ X_{(\min )}, & \text { otherwise. }\end{cases}
$$

Resource acquisition in this case for the sth SNO is obtained by solving the following optimization problem:

Problem 1:

$$
\operatorname{minimize}\left[\sum_{i_{j}=1}^{L} \sum_{k=1}^{N_{i_{j}}} c_{i_{j} k}(t) \cdot x_{i_{j} k}(t) \cdot \theta_{i_{j} k}(t)\right] \text {, }
$$

subject to

$$
\begin{aligned}
\underset{x_{i_{j}} \forall i, j, k}{\arg \min } \operatorname{Pr}\left(\lambda_{s i_{j}}(t), \mu_{s i_{j}}(t), \omega_{s i_{j}}\right) & \leq p_{s i_{j}}(t), \quad \forall i_{j}, k \\
x_{i_{j} k}(t) & \leq a_{i_{j} k}(t), \quad \forall i_{j}, k \\
\sum_{k=1}^{N_{i_{j}}} x_{i_{j} k}(t) & \leq r_{s i_{j}}(t), \quad \forall i_{j}, k
\end{aligned}
$$

where $\omega_{s i_{j}}=\sum_{k=1}^{N_{i_{j}}} x_{i_{j} k}(t)+w_{s i_{j}}$ is the total bandwidth (available and borrowed bandwidth from the PNOs).

In contrast to the formulation of Problem 1, borrowing cost for each cell $i$ can be calculated as $\sum_{k=1}^{N_{i_{j}}} c_{i_{j} k}(t) \cdot x_{i_{j} k}(t) \cdot \theta_{i_{j} k}(t)$. The parameter $\theta_{i_{j} k}(t)\left(0 \leq \theta_{i_{j} k}(t) \leq 1\right)$ defines the intrinsic quality by weighing the cost of borrowing spectrum bandwidths. The intrinsic quality represents the quality of the available heterogeneous aggregated channels to carry the data for transmission. Therefore, the price per unit bandwidth in each PNO can vary, i.e., $c_{i_{j} k}(t) \lesseqgtr c_{i_{j} l}(t), \forall i_{j}$ and $\forall k, l$ with $k \neq l$. We thus refer to this pricing scheme as non-uniform pricing.

The constraint (4) in Problem 1 guarantees that the sth SNO is borrowing enough to fulfil its demand. The blocking probability in constraint (4) is a non-linear function of spectrum bandwidth for each cell. Therefore, the above optimization problem is considered as a nonlinear optimization, which can be solved in two phases, In the first phase the SNOs set the target blocking probability for each cell (e.g., $p_{s i_{j}}=0.01, \forall i_{j}$ ). Then SNOs calculates the bandwidth $r_{s i_{j}}(t)$ required to achieve the target blocking probability $p_{s i_{j}}(t)$ for each cell $i$. Next the SNO finds the amount of bandwidth required to borrow from primary networks. We assume 
that the channel request rates and service rates follow Markov processes (i.e., inter-arrival and service times are exponential) for all PNOs and SNOs. A channel request is immediately lost if it finds the system busy, which implies that networks operate independently in a non-cooperative way. This is referred to as an Erlang loss system [25,34]. Under a loss system the well-known blocking probability for the $j$ th type of service at the $i$ th cell of the $s$ th SNO can be given by the Erlang B formula as

$$
p_{s i_{j}}(t)=\frac{1}{w_{s i_{j}} !}\left(\frac{\lambda_{s i_{j}}(t)}{\mu_{s i_{j}}(t)}\right)^{w_{s i_{j}}}\left[\sum_{n=0}^{w_{s i_{j}}} \frac{1}{n !}\left(\frac{\lambda_{s i_{j}}(t)}{\mu_{s i_{j}}(t)}\right)^{n}\right]^{-1}
$$

where $\lambda_{s i_{j}}(t), \mu_{s i_{j}}(t)$ and $w_{s i_{j}}$ are arrival rate, service rate and existing capacity of the $s$ th SNO, respectively. Note that during the post-optimization analysis new blocking probability formula are developed to accommodate sharing and interaction between operators in Section III-G.

Now with the existing bandwidth $w_{s i_{j}}$, we first calculate the total required bandwidth $\tau_{s i_{j}}(t)$ to achieve the target blocking probability for the $i$ th cell of the SNO

$$
\tau_{s i_{j}}(t)=f^{-1}\left(\operatorname{Pr}\left(\lambda_{s i_{j}}(t), \mu_{s i_{j}}(t), w_{s i_{j}}\right)\right)
$$

where $f^{-1}(\cdot)$ is the inverse function of $P_{(b)}(t)$ (equation 7) used to derive the required capacity over the existing capacity. As the function is non-linear in $\lambda_{s i_{j}}(t), \mu_{s i_{j}}(t)$ and $\tau_{s i_{j}}(t)$, it is not easy to get an explicit expression for $\tau_{s i_{j}}(t)$ for a given target blocking probability. However, it is possible to calculate $\tau_{s i_{j}}(t)$ iteratively for given values of $\lambda_{s i_{j}}(t), \mu_{s i_{j}}(t)$ and a target blocking probability $p_{s i_{j}}(t)$. Subtracting the existing bandwidth $w_{s i_{j}}$ from the total required $\tau_{s i_{j}}(t)$, we obtain the required bandwidth $r_{s i j}(t)$ at the $i$ th cell of the SNO during time interval $t$

$$
r_{s i_{j}}(t)=\tau_{s i_{j}}(t)-w_{s i_{j}}
$$

Now the problem is to find the feasible set of bandwidth $x_{i_{j} k}(t)$ from the PNOs which minimizes the borrowing cost. This is done in the next mathematical programming phase (see Algorithm 1 for details).

In the second phase, we set up the borrowing cost $c_{i_{j} k}(t)$ and the maximum possible bandwidth available $a_{i j}(t)$. The borrowing decisions of the $s$ th SNO are made subject to the lowest price from the set $\left\{a_{i_{j} k}(t)\right\}$. The decision variable $x_{i_{j} k}(t)$ in this context can be a combination of 
a number of acquisitions, e.g., the sth SNO selects the lowest price from the available set of bandwidths from the PNOs. If the acquired resources $a_{i_{j} k}(t)$ are insufficient to reach the target blocking probability $p_{s i_{j}}(t)$ (i.e., $r_{s i_{j} k}(t)-a_{i_{j} k}(t)>0$ ), then the SNO borrows from the remaining bandwidths from the set $\left\{a_{i_{j} 1}(t), a_{i_{j} 2}(t), \ldots, a_{i_{j} N}(t)\right\} \not \nexists a_{i_{j} k}(t)$ for which the cost is minimum. If the required blocking probability $p_{i_{j}}(t)$ is reached, then the SNO stops acquiring new spectrum bandwidths until the next time interval $(t+1)$.

Once the problem is solved, the new blocking probability for the sth SNO can be calculated as

$$
\begin{aligned}
P_{\left(b_{s j_{j}}^{n e w}\right)}(t) & =\operatorname{Pr}\left(\lambda_{s i_{j}}(t), \mu_{s i_{j}}(t),\left(w_{s i_{j}}+\sum_{k=1}^{N_{i_{j}}} x_{i_{j} k}(t)\right)\right) \\
& =\frac{1}{\omega_{s i_{j}} !}\left(\frac{\lambda_{s i_{j}}(t)}{\mu_{s i_{j}}(t)}\right)^{\omega_{s i_{j}}}\left[\sum_{n=0}^{\omega_{s i_{j}}} \frac{1}{n !}\left(\frac{\lambda_{s i_{j}}(t)}{\mu_{s i_{j}}(t)}\right)^{n}\right]^{-1}
\end{aligned}
$$

where

$$
\omega_{s i_{j}}=w_{s i_{j}}+\sum_{k=1}^{N_{i_{j}}} x_{i_{j} k}(t) \text {. }
$$

Consequently, the sth SNO will achieve the blocking probability with the required amount of bandwidths satisfying the target blocking probability $p_{s i_{j}}(t)$ or with the highest possible borrowed bandwidths which is mathematically expressed as

$$
P_{\left(b_{s i_{j}}^{\text {new }}\right)}(t)= \begin{cases}p_{s i_{j}}(t), & \sum_{k=1}^{N_{i_{j}}} a_{i_{j} k}(t) \geq r_{s i_{j}}(t) \\ P_{\left(b_{s i_{j}}^{\text {new }}\right)}(t), & \text { otherwise. }\end{cases}
$$

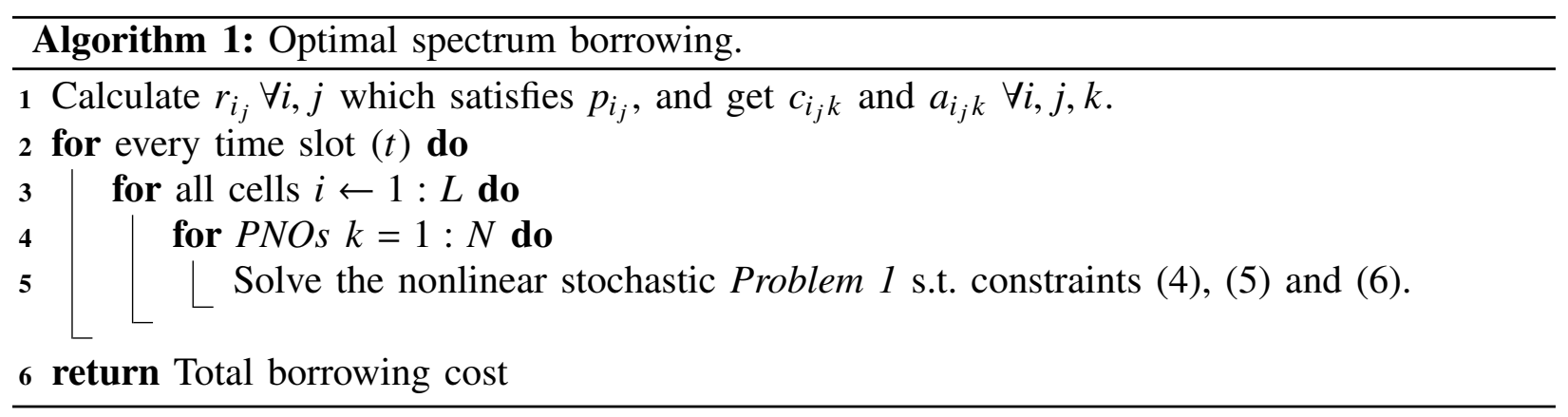




\section{Spectrum allocation using heuristic algorithm}

In this approach, spectrum acquisition is performed randomly as illustrated in Algorithm 2. The optimal borrowing cost using this algorithm can only be found randomly from the set of capacity values $\left\{a_{i_{j} k}\right\}$ by satisfying the constraints in equation (5) and (6).

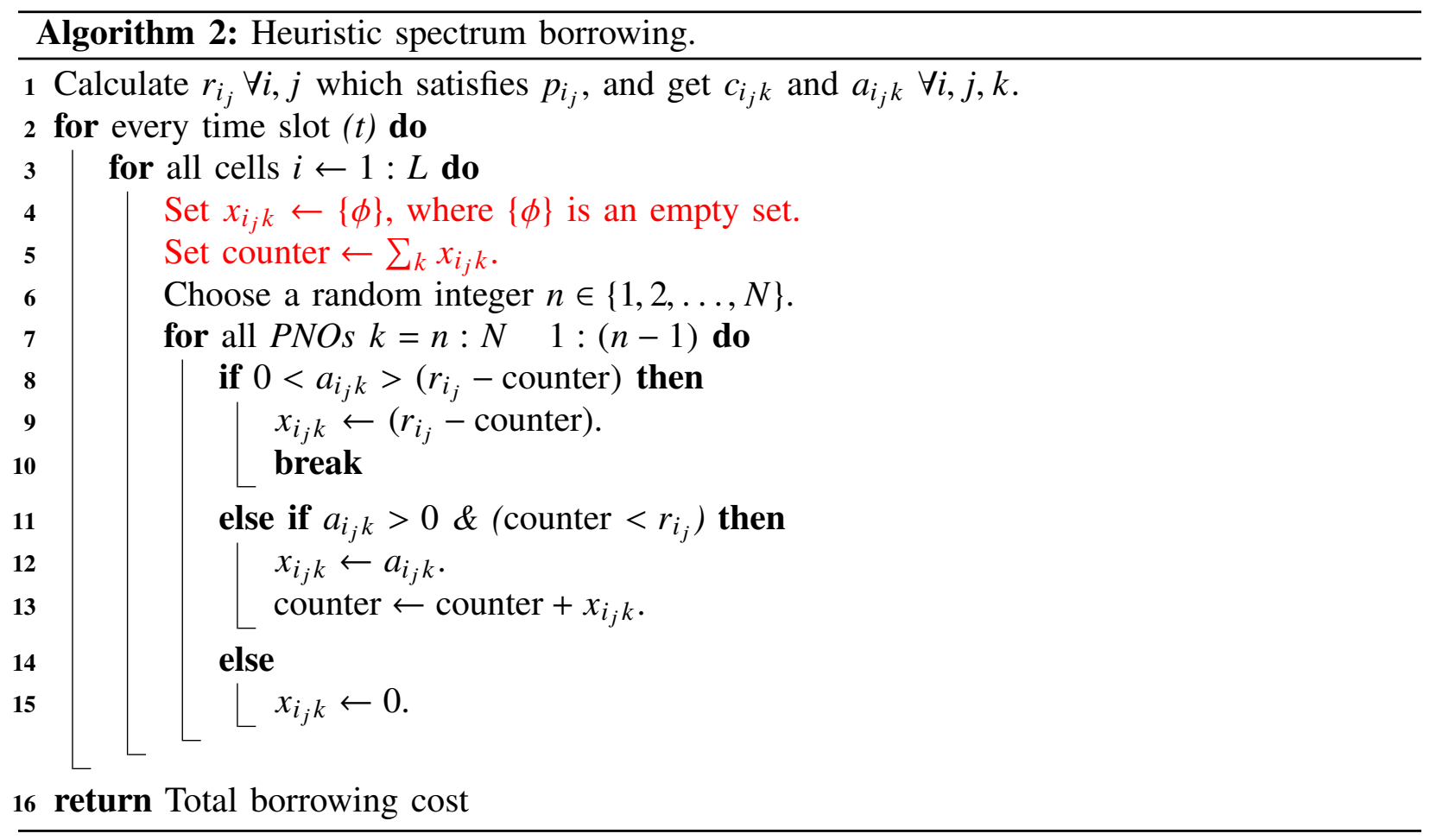

For all $i, j$ and $k$, equation (6) ensures that the sth SNO does not borrow more than the network's bandwidths demand by controlling the borrowed spectrum bandwidth size in each iteration, which can be expressed mathematically as

$$
x_{i_{j} k}(t)= \begin{cases}a_{i_{j} k}(t), & r_{i_{j}}(t) \geq a_{i_{j} k}(t) \\ r_{s i_{j}}(t), & \text { otherwise. }\end{cases}
$$

This scenario can also be regarded as round-robin scheduling algorithm, where SNOs randomly gain access to the PNOs' available spectrum, and the PNOs serve one SNO in each turn. The resource allocation in algorithm 2 evolves in two main discrete steps:

- compute the spectrum demand in each cell $r_{s i}, \forall i, j$ from equation (9)

- randomly obtain $x_{i_{j} k}$ subject to equations (5) and (6) from the vector $a_{i_{j} k}$ 
The only difference between the two formulations is that in the heuristic formulation, the cost of spectrum access is not considered, where spectrum acquisition is performed randomly from the set $\left\{a_{i_{j} k}\right\}$. Note that when $\sum a_{i_{j} k} \leq r_{s i_{j}}$ the feasible set $\left\{x_{i_{j} k}\right\}$ is equal for both formulations. We also note that when $\sum_{k=1}^{N_{i_{j}}} a_{i_{j} k}(t)>r_{s i_{j}}(t)$, the optimal and heuristic algorithm may achieve the same outcome in terms of total borrowing cost, however, this is a result of randomness in the selection process with probability

$$
P(\text { selecting optimal bandwidths })= \begin{cases}\frac{1}{N} & a_{i_{j} k} \geq r_{s i_{j}}, \forall i_{j} \\ \frac{1}{\mid\left\{\bar{a}_{i_{j} . .} \mid\right.} & \sum_{m}\left\{\bar{a}_{i_{j} l m}, \forall l, m\right\} \geq r_{s i_{j}}, \forall i_{j} \\ 1 & \sum_{k=1}^{N_{i_{j}}} a_{i_{j} k} \leq r_{s i_{j}}, \forall i_{j}\end{cases}
$$

where $\left\{\bar{a}_{i_{j} l m}, \forall l, m\right\} \subset\left\{a_{i_{j} k, \forall i_{j}, k}\right\}$, and $\left|\left\{\bar{a}_{i_{j} . .}\right\}\right|$ is the number of subsets in the set $\left\{\bar{a}_{i_{j} . .}\right\}$ which satisfy the bandwidth requirement for the $i$ th cell with $j$ th type of spectrum band.

Remark 1. In Problem 1, the objective function and all constraints are linear except for the constraint (4). Once we calculate the required bandwidth for the ith cell using the non-linear constraint (4) iteratively we then solve the optimization Problem 1 using Algorithm 1. With the remaining of the constraints the optimization problem is solved by the well-known revised simplex method. However, the computational complexity in Algorithm 1 is polynomial time, i.e. $O(n)$ time. The computational time increases linearly with number of cells and number of PNOs. The heuristic counterpart, Algorithm 2, arbitrarily borrows bandwidths from the PNOs until the target blocking probability of the SNO is achieved. Since the algorithm finds a solution by performing a combinatorics satisfying a set of constraints, the computational complexity is quadratic time, i.e. $O\left(n^{2}\right)$ with number of PNOs $(N)$ and exponential time, i.e. $2^{n}$ with number of cells (L). Note that the Algorithm 2 does not guarantee the optimal solution and the probability of finding an optimal solution by the heuristic algorithm is given in equation (14).

\section{E. Expected profit maximization under restricted budget}

In this section, we formulate the second spectrum allocation problem that illustrates how much spectrum bandwidths to be borrowed from each PNO to keep the blocking probability in 
a specific level. Given a set of possible available spectrum resources $\left\{a_{i_{j} k}(t)\right\}$, their associated prices $\left\{c_{i_{j} k}(t)\right\}$ and expected profit $\left\{\gamma_{i_{j} k}(t)\right\}$, the problem is to find the feasible set of spectrum bandwidths $\left\{x_{i_{j} k}(t)\right\}$ by maximising the total profit of the $s$ th SNO, under allocated budget and performing the selection process according to the highest possible profit combination. Resource acquisition in this case is obtained by solving the following optimization problem:

Problem 2:

$$
\operatorname{maximize}\left[\sum_{i=1}^{L} \sum_{j=1}^{M} \sum_{k=1}^{N_{j}} \gamma_{i_{j} k}(t) \cdot x_{i_{j} k}(t)\right]
$$

subject to

$$
\begin{aligned}
\underset{x_{i_{j} k} \forall i, j, k}{\arg \min } \operatorname{Pr}\left(\lambda_{s i_{j}}(t), \mu_{s i_{j}}(t), \omega_{s i_{j}}\right) & \leq p_{s i_{j}}(t), \quad \forall i_{j}, k \\
x_{i_{j} k}(t) & \leq a_{i_{j} k}(t), \forall i_{j}, k \\
\sum_{k=1}^{N_{i_{j}}} x_{i_{j} k}(t) & \leq r_{s i_{j}}(t), \forall i_{j}, k \\
\sum_{k=1}^{N_{j}} c_{i_{j} k}(t) \cdot x_{i_{j} k}(t) & \leq b_{s i_{j}}, \forall s, i_{j}, k,
\end{aligned}
$$

where $\gamma_{i_{j} k}(t)$ consists of two parts: the expected revenue $v_{i_{j}}(t)$ and cost $c_{i_{j} k}(t)$, which can be obtained as

$$
\gamma_{i_{j} k}(t)=v_{i_{j} k}(t)-c_{i_{j} k}(t)
$$

Here

$$
v_{i_{j} k}(t)=f\left(\beta_{i_{j}}(t), \theta_{i_{j} k}(t)\right)
$$

where $\beta_{i_{j}}(t)$ is the selling price per unit bandwidth for the $i$ th cell and $j$ th type service during time period $t$. In equation (21), the expected revenue $v_{i_{j} k}(t)$ is the function $f(\cdot)$ of the selling price $\beta_{i_{j} k}(t)$ and the intrinsic quality $\left(\theta_{i_{j} k}(t)\right)$ which may take, in general, a non-linear form. In the simplest case, the function can be defined as

$$
v_{i_{j} k}(t)=\beta_{i_{j} k}(t)\left[-e^{-\theta_{i_{j} k}(t)}\right] .
$$

We consider the the intrinsic quality per unit bandwidth $\left(\theta_{i_{j} k}(t)\right)$ for each PNO, which can vary, i.e., $\theta_{i_{j} k}(t) \lesseqgtr \theta_{i_{j} l}(t), \forall i_{j}$ and $\forall k, l$ with $k \neq l$ according to spatial structure of the base stations, allowed transmission power, bandwith types, etc. In this problem formulation, the parameter 
$\theta_{i_{j} k}(t)$ influences the optimal spectrum borrowing decisions.

The revenue earned through the sale of the borrowed bandwidth can be equal, higher or lower than the cost. However, for simplicity, we model the revenue $v_{i_{j} k}(t)$ earned through the sale of the borrowed bandwidth to exceed the borrowing cost, i.e., $v_{i_{j} k}(t)>c_{i_{j} k}(t)$ due to the assumption that profit of the $s$ th SNO for borrowing a unit bandwidth is always positive $\left(\gamma_{i_{j} k}(t)\right)$.

The inequality constraint in equation (19) implies that the sth SNO maximizes its profit by taking into account the limitations imposed by cost of the utility and the maximum allowable expenditure which the sth SNO can spend for borrowing spectrum demand in each cell. Next, we solve the the above non-linear optimization problem in two phases:

In the first phase, the same steps are performed using equation (16) as for solving Problem 1. The $s$ th SNO calculates the spectrum demand to meet its time varying target blocking probability over time and location. The spectrum demand is adjusted dynamically based on the network information provided by the expected cell demand, service rate and existing spectrum bandwidth.

In the second phase, we set up the vectors $\left\{c_{i_{j} k}(t)\right\},\left\{a_{i_{j} k}(t)\right\}$ and $\left\{\gamma_{i_{j} k}(t)\right\}$. The borrowing decisions of the sth SNO are made subject to achieving the maximum profit for each acquisition from the PNOs. In Problem 1, the budget restriction is not considered, where the sth SNO is allowed to make spectrum bandwidth borrowing until it meets the spectrum demand, i.e.,

$$
\sum_{k=1}^{N_{j}} x_{i_{j} k}(t)=r_{s i_{j}}(t) \text {, assuming } \sum_{k=1}^{N_{j}} a_{i_{j} k}(t) \geq r_{s i_{j}}(t) \text {. }
$$

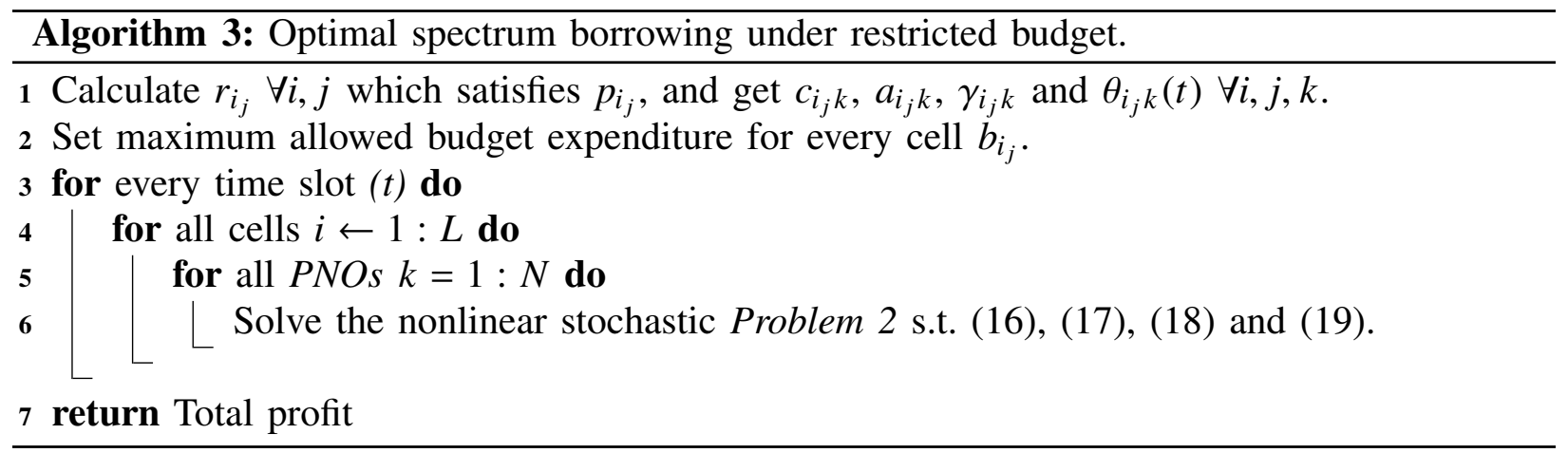

However, in this formulation, the borrowing capacity of each SNO is restricted to budget allocation $b_{s i_{j}}$. Note that in the case where an SNO's budget is too small to provide the required 
GoS, then Problem 2 is infeasible. The SNOs only achieves a best effort service to minimize the blocking probability so far as the budget permits.

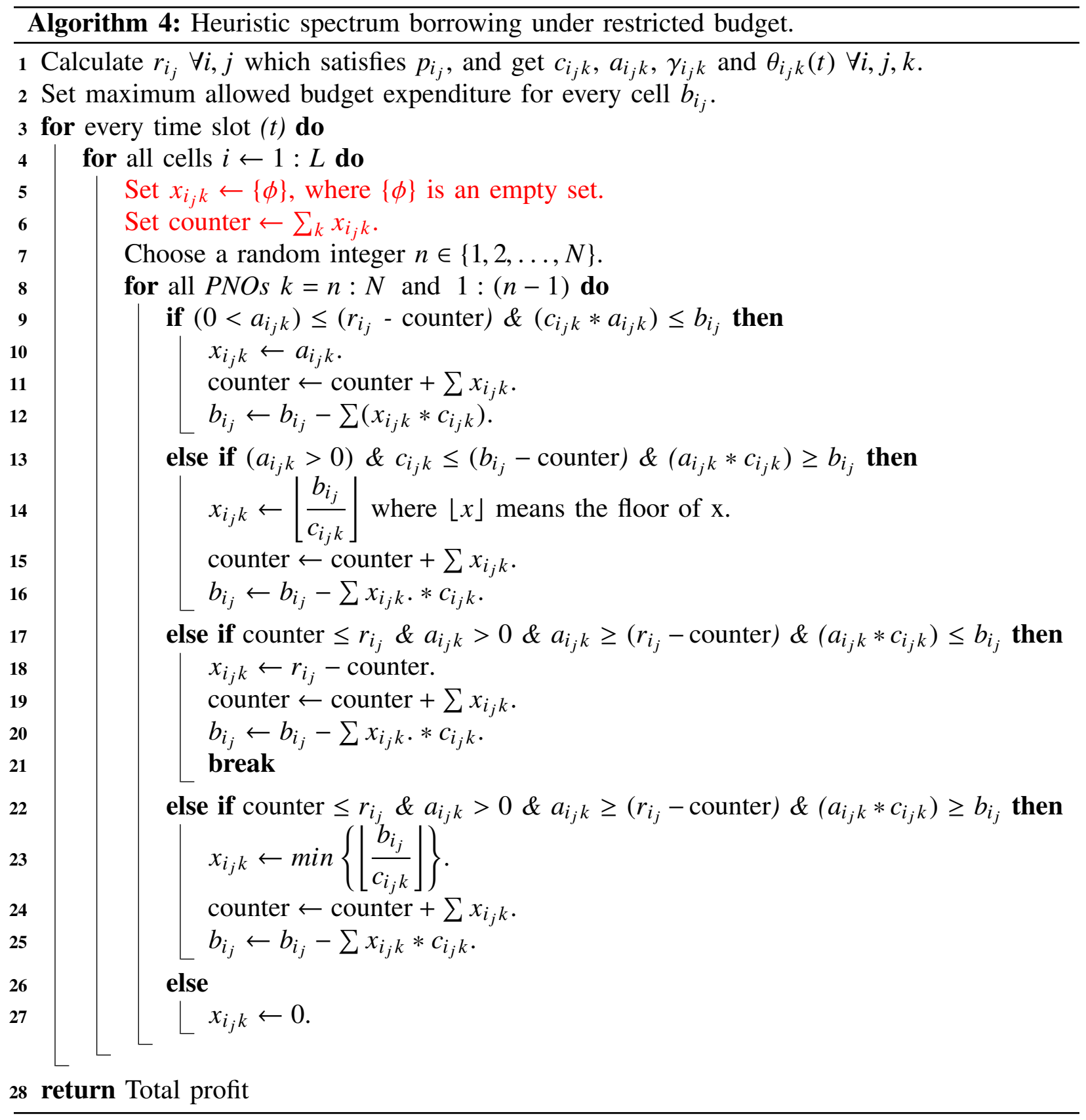

\section{F. Spectrum allocation using heuristic algorithm under budget constraint}

In this subsection, we solve the problem of spectrum allocation under budget constraint by a heuristic bandwidth selection algorithm (Algorithm 4). The algorithm performs all the steps 
as in Algorithm 3. However, Algorithm 4 does not perform spectrum selection according to the highest possible profit combination from the set $\left\{a_{i_{j}}\right\}$, rather runs on randomly selected combination from the set $\left\{a_{i_{j} k}\right\}$ to satisfy the spectrum demand $r_{s i_{j}}$. The optimal profit using Algorithm 4 can only be found from the set of capacity values $\left\{a_{i_{j} k}\right\}$ satisfying the constraints in equation (17), (18) and (19) with probability given in equation (14). To satisfy the constraints in equation (17), (18) and (19) we use

$$
x_{i_{j} k}(t)= \begin{cases}a_{i_{j} k}(t), & r_{s i_{j}}(t) \geq a_{i_{j} k}(t), b_{s i_{j}} \geq c_{i_{j} k}, \\ r_{s i_{j}}(t), & r_{s i_{j}}(t)<a_{i_{j} k}(t), b_{s i_{j}} \geq c_{i_{j} k}, \\ 0, & b_{s i_{j}}<c_{i_{j} k} \text { or } r_{s i_{j}}(t)=0 .\end{cases}
$$

Remark 2. Like Problem 1, in Problem 2 we have the non-linear constraint which is solved iteratively and then the whole problem is solved by the revised simplex method. Therefore, Algorithm 3 is polynomial time and the heuristic counterpart (Algorithm 4) is again quadratic timelexponential time depending on number of cells and PNOs.

\section{G. Post-optimization analysis under resource sharing between SNOs and PNOs}

In the optimization problems above, the PNOs lease part of their spectrum resources to SNOs for monetary benefits. The leasing and borrowing was based on fixed arrival rate and available spectrum resources. However, the demand in the PNOs may be bursty during trading window causing one or more of PNOs' state to change from the underloaded to overloaded and their blocking probability would increase. As a consequence, a PNO may react by deviating part or all of its leased spectrum resources under mutual agreement, which results in reducing the size of the shared spectrum resources. This dynamic mechanism affects the performance of all operators involved in the trading. The complexity of the problem depends primarily on the number of PNOs and SNOs involved and the level of interactions between them.

In this paper, we present an analytical methodology to model and analyze the above consequences in cellular networks with multiple primary and secondary networks. 
Consider a cell consisting of $S$ SNOs with capacity $c_{s_{1}}, c_{S_{2}}, \ldots, c_{S_{S}}$ and $N$ PNOs with capacity $c_{1}, c_{2}, \ldots, c_{N}$, respectively. Under a sharing agreement all $N$ PNOs share part of their resources $c_{1}^{\prime}, c_{2}^{\prime}, \ldots, c_{N}^{\prime}$, respectively with $S$ SNOs determined using the optimization approach discussed in the previous sections. These resources may also be used by the corresponding PNO under mutual agreement. A state of the network is a vector of length $[S(1+N)+2 N)]$ defined as

$$
\begin{aligned}
\mathbf{n}=\left\{\left(n_{s_{1}}, n_{s_{2}}, \ldots, n_{s_{s}}\right) ;\left(\left(n_{s_{1}} 1, n_{s_{1}}, \ldots, n_{s_{1} n}\right), \ldots,\left(n_{s_{S} 1}, n_{s_{S} 2}, \ldots, n_{s_{S} n}\right)\right) ;\right. \\
\left.\quad\left(n_{1}, n_{2}, \ldots, n_{N}\right) ;\left(n_{11}, n_{22}, \ldots, n_{N N}\right)\right\}
\end{aligned}
$$

with the condition that $\left(n_{s_{1}}, n_{s_{2}}, \ldots, n_{s_{S}}\right)+n_{i i} \leq c_{i}^{\prime} \forall i=1,2, \ldots, N$, where $n_{s_{i}}$ is the number of channel requests in progress in the $i$ th $\mathrm{SNO} ; n_{s_{i} j}$ is the number of channel requests in the shared resources of the $i$ th PNO from the $s_{i}$ th $\mathrm{SNO} ; n_{j}$ is the number of channel requests in progress in the $j$ th PNO and $n_{j j}$ is the number of request of the $j$ th PNO on its own shared resources. The states $\mathbf{n}$ are restricted due to resource sharing constraints. The set of feasible states can be written as

$$
\Omega_{s}=\{\mathbf{n}: \mathbf{A n} \leq \mathbf{s}\}
$$

where $\mathbf{A}$ is a $[S(1+N)+2 N)]$ matrix and $\mathbf{s}$ is a $d$-vector, where $d$ is the number of constraints. The network topology is reflected in the matrix $\mathbf{A}$ and the vector $\mathbf{s}$.

Let channel requests arrive to the $i$ th secondary and $j$ th primary operators according to a nonhomogeneous Poisson process, with rates $\lambda_{s_{i}}(t)$ and $\lambda_{j}(t)$ at time $t$. These requests are admitted if $\mathbf{n}+\mathbf{e}_{i} \in \Omega_{s}$ (if PNO) and $\mathbf{n}+\mathbf{e}_{j} \in \Omega_{s}$ (if SNO), where $\mathbf{e}_{i}$ and $\mathbf{e}_{j}$ is the $i$ th unit vector with 1 in place $i$ and 0 elsewhere. When all $c_{s_{i}}$ resources of the $i$ th SNO is full then requests are diverted and admitted to the $j$ th PNO if $\mathbf{n}+\mathbf{e}_{i j} \in \Omega_{s}$, where $\mathbf{e}_{i j}$ is the $i$ th unit vector. Similarly, in the $j$ th PNO being all $c_{j}$ resources occupied requests are diverted to its shared resources $c_{j}^{\prime}$ for the $j$ th primary network if $\mathbf{n}+\mathbf{e}_{j j} \in \Omega_{s}$, where $\mathbf{e}_{j j}$ is the $i$ th unit vector. Assume that admitted requests in $i$ th secondary and $j$ th primary operators have exponential holding times with rates $\mu_{s_{i}}(t)$ and $\mu_{j}(t)$ respectively at time $t$. Under these assumptions, the network can be modeled as a non-homogeneous continuous time Markov chain

$$
\mathbf{X}(t)=\left(X_{i}(t), X_{i j}(t), X_{j j}(t), X_{j}(t) ; i=1,2, \ldots, S, j=1,2, \ldots, N, t \geq 0\right)
$$

that records the number of channel requests in progress from all operators. The state space of 
the Markov chain is specified in (25), and its transition rates $\mathbf{Q}(t)=\left(q\left(\mathbf{n}, \mathbf{n}^{\prime}, t\right), \mathbf{n}, \mathbf{n}^{\prime} \in \Omega_{s}\right)$ are given by

$$
q\left(\mathbf{n}, \mathbf{n}^{\prime}, t\right)= \begin{cases}\lambda_{s_{i}}(t) & \mathbf{n}^{\prime}=\mathbf{n}+\mathbf{e}_{i} \text { or } \mathbf{n}^{\prime}=\mathbf{n}+\mathbf{e}_{i j}, \text { if } n_{s_{i}}=c_{s_{i}}, i=1,2, \ldots, S \\ \lambda_{j}(t) & \mathbf{n}^{\prime}=\mathbf{n}+\mathbf{e}_{j} \text { or } \mathbf{n}^{\prime}=\mathbf{n}+\mathbf{e}_{j j}, \text { if } n_{j}=c_{j}, j=1,2, \ldots, N \\ n_{s_{i}} \mu_{s_{i}}(t) & \mathbf{n}^{\prime}=\mathbf{n}-\mathbf{e}_{i}, i=1,2, \ldots, S \\ n_{j} \mu_{j}(t) & \mathbf{n}^{\prime}=\mathbf{n}-\mathbf{e}_{j}, j=1,2, \ldots, N \\ n_{s_{i j}} \mu_{s_{i}}(t) & \mathbf{n}^{\prime}=\mathbf{n}-\mathbf{e}_{i j}, i=1,2, \ldots, S, j=1,2, \ldots, N \\ n_{j j} \mu_{j}(t) & \mathbf{n}^{\prime}=\mathbf{n}-\mathbf{e}_{j j}, j=1,2, \ldots, N\end{cases}
$$

Theorem 1. The closed-form solution of $\mathbf{n}$ channel requests in progress at time $t$ is given by equation (28).

$$
\begin{gathered}
P(\mathbf{n}, t)=\mathcal{K}^{-1} \frac{\left[\rho_{s_{1}}^{\left(n_{s_{1}}+n_{s_{1} 1}+\cdots+n_{s_{1} N}\right)}(t)\right] \cdots\left[\rho_{s_{S}}^{\left(n_{S_{S}}+n_{S_{S}}+\cdots+n_{S_{S} N}\right)}(t)\right]}{\left(n_{S_{1}}+n_{s_{1} 1}+\cdots+n_{s_{1} N}\right) ! \cdots\left(n_{S_{1}}+n_{s_{1} 1}+\cdots+n_{s_{1} N}\right) !} \\
\cdot \frac{\left[\rho_{1}^{\left(n_{1}+n_{11}\right)}(t)\right] \cdot\left[\rho_{2}^{\left(n_{2}+n_{22}\right)}(t)\right] \cdots\left[\rho_{N}^{\left(n_{N}+n_{N N}\right)}(t)\right]}{\left(n_{1}+n_{11}\right) !\left(n_{2}+n_{22}\right) ! \cdots\left(n_{N}+n_{N N}\right) !} \forall \mathbf{n} \in \Omega_{S}
\end{gathered}
$$

where $\rho_{s_{i}}=\lambda_{s_{i}} / \mu_{s_{i}}$ and $\rho_{j}=\lambda_{j} / \mu_{j}$ are traffic intensities of the $s_{i}$ th SNO and $j$ th PNO, respectively and $\mathcal{K}$ is the normalizing constant and given by

$$
\begin{gathered}
\mathcal{K}=\sum_{\mathbf{n} \in \Omega_{s}} \frac{\left[\rho_{s_{1}}^{\left(n_{s_{1}}+n_{s_{1}}+\cdots+n_{s_{1} N}\right)}(t)\right] \cdots\left[\rho_{S_{S}}^{\left(n_{S_{S}}+n_{S_{S}}+\cdots+n_{S_{S} N}\right)}(t)\right]}{\left(n_{S_{1}}+n_{s_{1} 1}+\cdots+n_{s_{1} N}\right) ! \cdots\left(n_{s_{1}}+n_{s_{1} 1}+\cdots+n_{s_{1} N}\right) !} \\
\cdot \frac{\left[\rho_{1}^{\left(n_{1}+n_{11}\right)}(t)\right] \cdot\left[\rho_{2}^{\left(n_{2}+n_{22}\right)}(t)\right] \cdots\left[\rho_{N}^{\left(n_{N}+n_{N N}\right)}(t)\right]}{\left(n_{1}+n_{11}\right) !\left(n_{2}+n_{22}\right) ! \cdots\left(n_{N}+n_{N N}\right) !} \forall \mathbf{n} \in \Omega_{s} .
\end{gathered}
$$

Proof. Define the state probabilities

$$
P(\mathbf{n}, t):=\operatorname{Pr}(X(t)=\mathbf{n}), \quad \mathbf{n} \in \Omega_{s}, t \geq 0
$$

with initial condition $P_{0}(\mathbf{n})=\operatorname{Pr}(X(0)=\mathbf{n})$. Since the network has a finite state space, these probabilities are the unique solution of the Kolmogorov forward equations given in (31) for $\mathbf{n} \in \Omega_{s}, t>0$. 
The Kolmogorov forward equations can be defined as

$$
\begin{aligned}
& \frac{\mathrm{d} P(\mathbf{n}, t)}{\mathrm{d} t}=\left[\sum_{i=1}^{S} \lambda_{s_{i}}(t) \cdot\left(\mathbb{1}\left(n_{s_{i}}<c_{s_{i}}\right)+\mathbb{1}\left(n_{s_{i}}=c_{s_{i}} \cap \mathbf{n}+\mathbf{e}_{i j}\right)\right)+\sum_{j=1}^{N} \lambda_{j}(t) \cdot\left(\mathbb{1}\left(n_{j}<c_{j}\right)\right.\right. \\
&\left.\left.+\mathbb{1}\left(n_{j}=c_{j} \cap \mathbf{n}-\mathbf{e}_{j j}\right)\right)\right] \cdot P\left(\left(\mathbf{n}-\mathbf{e}_{i}\right), t\right)+\sum_{i=1}^{S}\left(n_{s_{i}}+1\right) \mu_{s_{i}}(t) P\left(\left(\mathbf{n}+\mathbf{e}_{i}\right), t\right) \\
&+\sum_{j=1}^{N}\left(n_{j}+1\right) \mu_{j}(t) \cdot P\left(\left(\mathbf{n}+\mathbf{e}_{j}\right), t\right)-\left[\sum_{i=1}^{S} \lambda_{s_{i}}(t) \cdot\left(\mathbb{1}\left(n_{s_{i}}<c_{s_{i}}\right)+\mathbb{1}\left(n_{s_{i}}=c_{s_{i}} \cap \mathbf{n}+\mathbf{e}_{i j}\right)\right)\right. \\
&\left.+\sum_{j=1}^{N} \lambda_{j}(t) \cdot\left(\mathbb{1}\left(n_{j}<c_{j}\right)+\mathbb{1}\left(n_{j}=c_{j} \cap \mathbf{n}-\mathbf{e}_{j j}\right)+\sum_{i=1}^{S} n_{s_{i}} \mu_{s_{i}}(t)+\sum_{j=1}^{N} n_{j} \mu_{j}(t)\right)\right] P(\mathbf{n}, t)
\end{aligned}
$$

where $\mathbb{1}(A)$ is the indicator function for an event $A$.

Equations in (31) can be written in the operator form as given by

$$
\frac{\mathrm{d} \mathbf{P}(t)}{\mathrm{d} t}=\mathbf{P}(t) \mathbf{Q}(t), \quad \mathbf{P}(0)=\mathbf{P}_{0}, t>0
$$

where $\mathbf{P}(t)$ is the vector of probabilities $\mathbf{P}(\mathbf{n}, t)$. The formal solution of the equation (32) is given by

$$
\mathbf{P}(t)=\mathbf{P}_{0} E_{Q}(t), \quad t \geq 0
$$

where $E_{Q}(t)$ is the time-ordered exponential of the generator $\mathbf{Q}(t)$, that is the unique operator solution to the equation

$$
\frac{\mathrm{d} E_{Q}(t)}{\mathrm{d} t}=E_{Q}(0) \mathbf{Q}(t), \quad t \geq 0
$$

where $E_{Q}(0)=\mathbf{I}$, the identity operator. The unique solution of the Kolmogorov forward equations (31) is then given by the equation (28).

The blocking probability formula can then be derived from the closed-form solution (28). The blocking probability for an operator $i$ (ith operator could be an SNO or a PNO), is then given by

$$
P_{b}(t)=\sum_{\mathbf{n} \in \mathcal{S}} P(\mathbf{n}, t)
$$




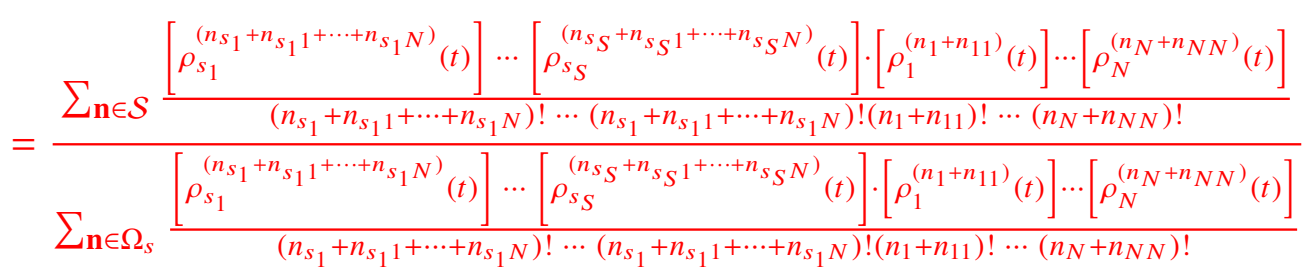

where the set $\mathcal{S}$ is the restricted state space, and varies for the SNOs and PNOs. For the $s$ th SNO, it is defined as

$\mathcal{S}_{i}=\left\{\mathbf{n} \in \Omega_{s} \mid\left(n_{s_{i}}=c_{s_{i}} \cap n_{s_{i} 1}+n_{11}=c_{1}^{\prime} \cap n_{s_{i}}+n_{22}=c_{2}^{\prime} \cap \cdots \cap n_{s_{i} N}+n_{N N}=c_{N}^{\prime}\right)\right\}, i=1,2, \ldots, S$.

and for the $j$ th $\mathrm{PNO}, \mathcal{S}_{i}$ can be replaced by $\mathcal{S}_{j}$ and defined as

$$
\mathcal{S}_{i}=\left\{\mathbf{n} \in \Omega_{s} \mid\left(n_{s_{i}}=c_{s_{i}} \cap n_{s_{i} j}+n_{j j}=c_{i}^{\prime}\right)\right\}, j=1,2, \ldots, N
$$

\section{RESULTS AND ANALYSIS}

In this section, we show the analysis of optimal borrowing solutions by Algorithms 1 and 3 corresponding to the cost minimization and profit maximization with restricted budget scenarios, respectively. To explore the advantages of the proposed formulations, we compare the results from Algorithm 1 and 3 with a heuristic spectrum selection formulation by Algorithm 2 and 4, respectively. We simulate the functionalities of the network management, which are necessary to generate the optimal solution and to compare with the heuristic spectrum selection algorithms. We consider one SNO and four PNOs $(N=4)$ to simulate the dynamics of the merchant mode resource sharing mechanism. Some parameters are determined randomly by the algorithms with specific distribution (e.g., $\lambda_{i}, \mu_{i}, w_{i}$ ) while other parameters are preset (e.g., $L, p_{i_{j}}$ ).

TABLE I: Simulation parameters.

\begin{tabular}{lcccccccccc}
\hline Parameter & $L$ & $M$ & $N$ & $p_{i_{j}}$ & $\lambda_{i_{j}}$ & $\mu_{i_{j}}$ & $w_{i_{j}}$ & $c_{i_{j} k}$ & $a_{i_{j} k}$ & $b_{i_{j}}$ \\
\hline Values for problem 1 & 100 & 1 & 4 & 0.01 & 10 & 1 & 1 & $(3,9)$ & $(5,10)$ & -- \\
\hline Values for problem 2 & 100 & 1 & 4 & 0.01 & $(40,120)$ & $(1,5)$ & $(1,5)$ & $(10,13)$ & $(30,40)$ & 50 \\
\hline
\end{tabular}




\section{A. Cost analysis under target performance (Problem 1)}

In the simulation, we consider the PNOs spectral usage for all cells $L$, where four base stations of primary network operators in each cell are deployed (collocated topology), e.g., the case in densely populated cities. The demand of service for each provider (primary or secondary) vary over time and location. We assume the spectral utilisation of secondary provider at time interval $t$ is high whereas the PNOs are underloaded in the same time interval and at the same location.

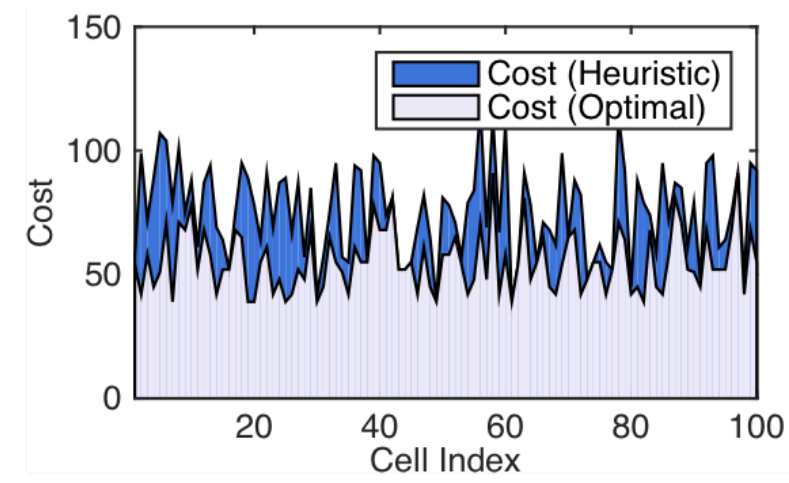

(a)

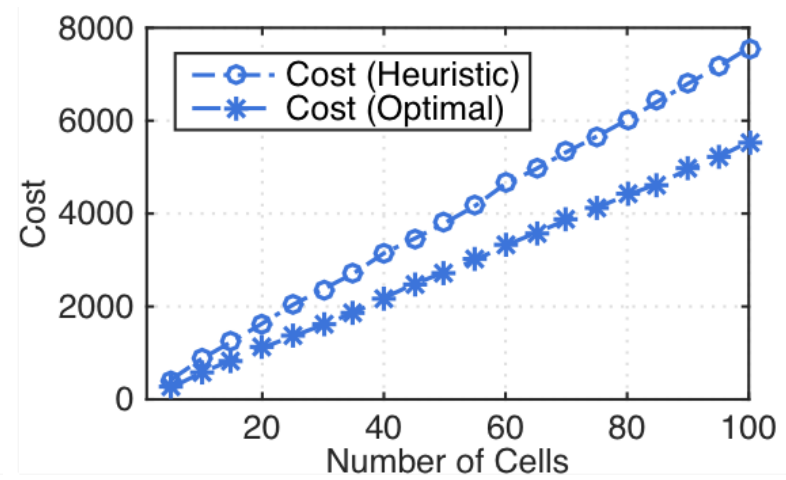

(b)

Fig. 2: Cost with optimal and heuristic algorithms for (a) per cell and (b) for varying number of cells.

The PNOs charge the SNO at variable rates. The charges may be assessed by the market on the basis of the current supply-demand balance for each individual operator at each cell and possibly other factors [12]. However, we set limits to the price of unit bandwidth as maximum $X_{(\max )}$ and minimum price $X_{(\min )}$ to structure the problem space. For the purpose of analysis, we parametrise the borrowing cost as $c_{i_{j} k}(t)=\left\{c_{i_{j} k}(t) \mid X_{(\min )} \leq c_{i_{j} k}(t) \leq X_{(\max )}\right\}$, where $c_{i_{j} k}(t)$ follows a uniform distribution from $\left[X_{(\min )}=3, X_{(\max )}=9\right]$. We keep the difference between $X_{(\max )}$ and $X_{(\min )}$ relatively small at all cells. This assumption captures the highly competitive nature of the market economic environment. We determine the admission cost per unit bandwidth based on a discrete uniform random variables. In our mathematical model all possible variations of the available bandwidth values $a_{i_{j} k}(t)$ to provide the SNO demand are considered. This assumption provides realistic scenarios where PNOs could have different values of leasable spectrum resources. More details about the simulation parameters are given in Table I.

At time $t$ and in each cell $i$, the SNO has a particular blocking probability target $p_{i_{j}}$. By 
considering the SNO's expected traffic load $\lambda_{i_{j}}$ in the next time interval, the available capacity $w_{i_{j}}$ and service rate $\mu_{i_{j}}$, each cell determines its required number of channels $r_{i_{j}}(t)$.

For comparisons, we simulate the interactions between the network providers and we solve the resource allocation problem by the optimal and the heuristic allocation as described in Algorithms 1 and 2, respectively. For the simulation of the heuristic allocation, each cell $i$ makes heuristic selection of aggregated channels for dynamic access from the set $\left\{a_{i_{j} k}\right\}$ which are collocated in the same cell. The selection of aggregated channels is performed regardless of the admission cost associated with the choice of selected channels. Algorithm 2 is allowed to perform spectrum borrowing until the demand is satisfied, assuming $\sum_{k=1}^{N_{i_{j}}} a_{i_{j} k}(t) \geq r_{i_{j}}(t)$. If $\sum_{k=1}^{N_{i_{j}}} a_{i_{j} k}(t)<r_{i_{j}}(t)$ then the algorithm takes all available bandwidths, however, the target blocking probability will not be satisfied, such that, $P_{\left(b_{n e w}\right)}(t)<p_{i_{j}}(t)$.

For the Algorithm 1, the cells of SNO select the combination $\left\{x_{i_{j} k}\right\}$ with the lowest admission cost from the set $\left\{a_{i_{j} k}(t)\right\}, \forall k, i$, to achieve the optimal channel borrowing admission costs. It is possible that there may be multiple solutions for the allocation problem which provide the same required bandwidth to the SNO with different costs.

The main observation here is that the optimal model achieves lower costs compared to the heuristic algorithm, except for cells with $\sum_{k=1}^{N_{i_{j}}} a_{i_{j} k}(t)<r_{i_{j}}(t)$, see Figure $2 \mathrm{a}$. It is also observed that the total borrowing cost of both the heuristic and optimal configuration vary in every cell due to the stochastic nature of the costs and number of available channels.

If we consider the admission cost for large number of cells, as we can see from Figure $2 b$, we notice that as the number of cells increase, the difference in cost between the heuristic and the optimal selection algorithm becomes larger, which implies substantial savings for operators with large territories when the optimal algorithm is used.

We also investigate the effect of target blocking probability on the admission cost. In Figure 3 we show the results for different target blocking probabilities ranging from $0-0.9$ for a single cell. We clearly see that as $p_{i_{j}} \rightarrow 0$, the admission cost increases for both algorithms. However, the optimal algorithm (Algorithm 1) provides lower borrowing cost for most of the points.

The total number of aggregated channels which are acquired through borrowing by using Algorithms 1 and 2 is equal, see Figure 4. This is because both algorithms allow channels to be acquired until a certain GoS is reached or until all channels from the available bandwidths 


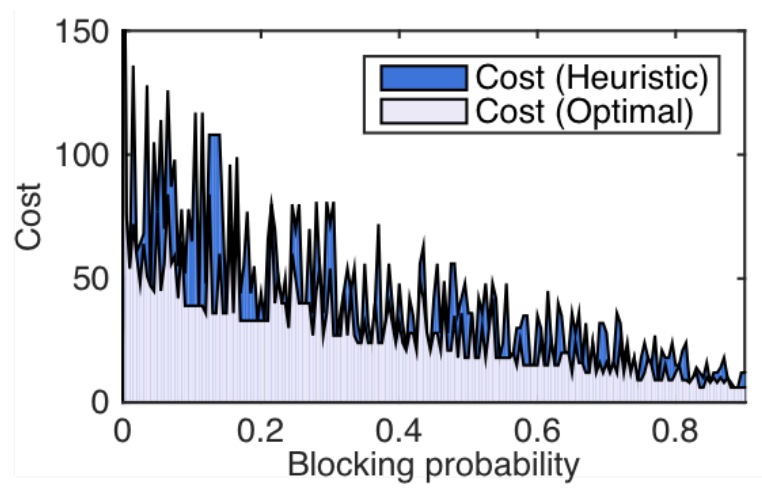

Fig. 3: Effect of varying target blocking probability on cost for optimal and heuristic algorithms.

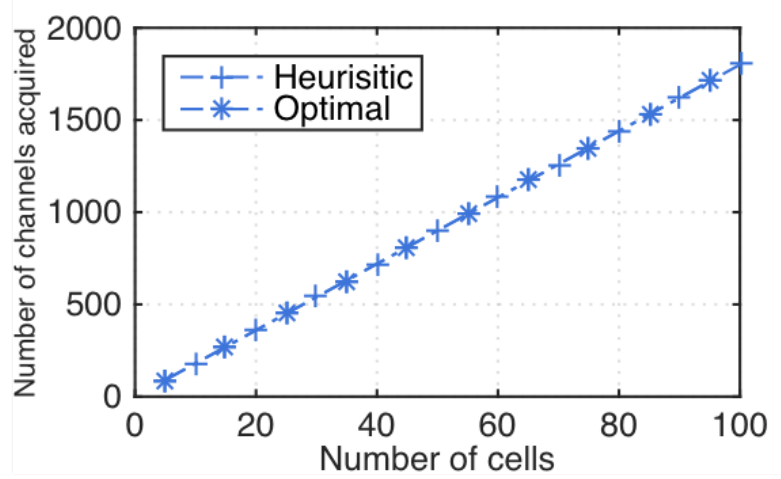

Fig. 4: Effect of borrowing on bandwidth acquisition for the optimal and heuristic algorithms.

of PNOs $\left\{a_{i_{j} k}\right\}$ are consumed.

\section{B. Expected profit analysis under budget constraints (Problem 2)}

The objective of the secondary providers can be described from both economic and system performance perspective. Firstly, the SNO aims to lower the blocking probability for its subscribers. Secondly, the SNO attempts to maximize its profit by leasing additional spectrum from the PNOs in terms of cost and intrinsic quality. However, since network operators often operate with limited budget, SNO can only spend $b_{i_{j}}(t)$ amount of resources/money at a cell $i$ and time interval $t$. This is imposed by the government and regulatory bodies to keep the fairness of spectrum leasing among network operators.

To demonstrate the gain of Algorithm 3, detailed investigation has been made and the results are compared with Algorithm 4. Figure 5a shows the optimal algorithm can achieve substantial gain in comparison to the heuristic allocation approach. However, both algorithms provide acceptable efficiency in terms of GoS. We also notice that as the number of cells increase the profit of the SNO gets larger, see Figure $5 \mathrm{~b}$.

Figure 6 shows the effect of budget and target blocking probability on achievable profit with varying budget expenditure between $0-250$ and target blocking probability between 0 and 0.8 for a single cell. It is clear that as we increase the budget further $b_{i_{j}} \rightarrow 250$, the profit increases with respect to the increase of budget and demand. However, as the budget reaches a certain value, the profit does not increase because the budget is larger than required. 


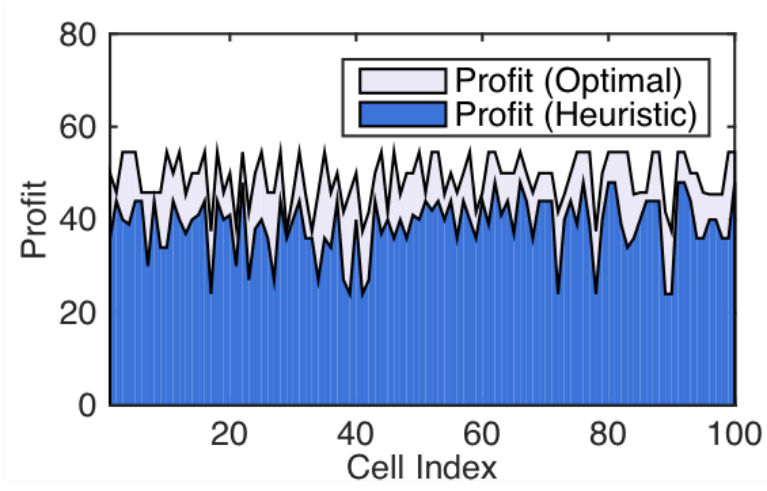

(a)

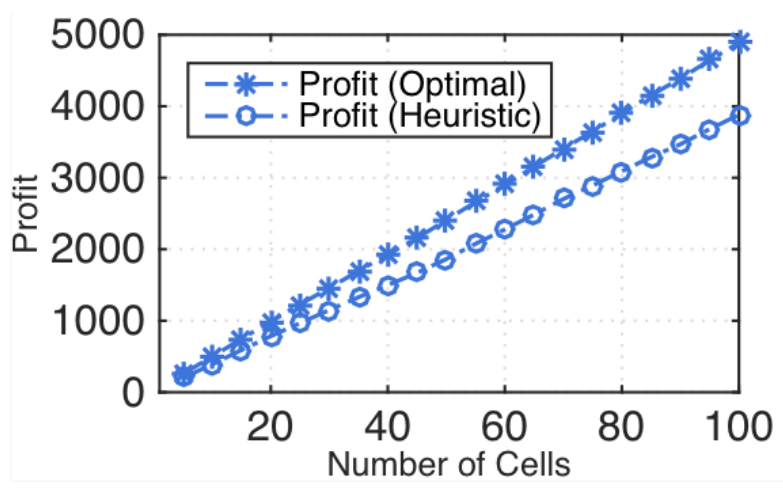

(b)

Fig. 5: Profit using the optimal and heuristic algorithms for (a) per cell and (b) for varying number of cells.

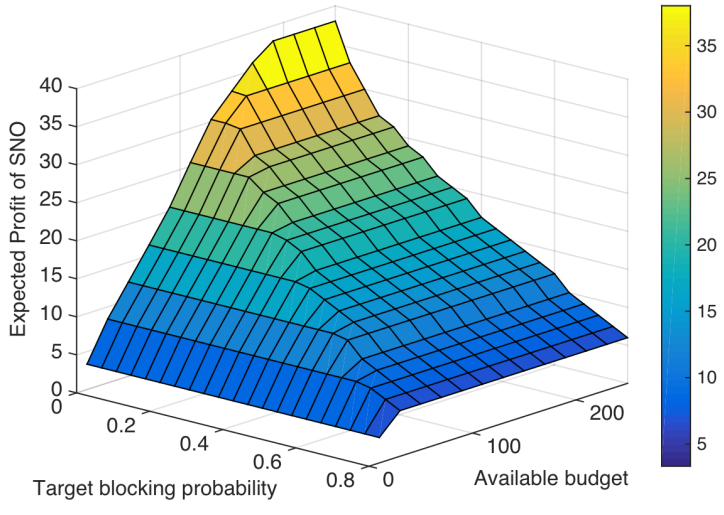

Fig. 6: Expected profit of the SNO for spectrum borrowing with target blocking probability $=0$ to 0.8 and budget $=0$ to 250 .

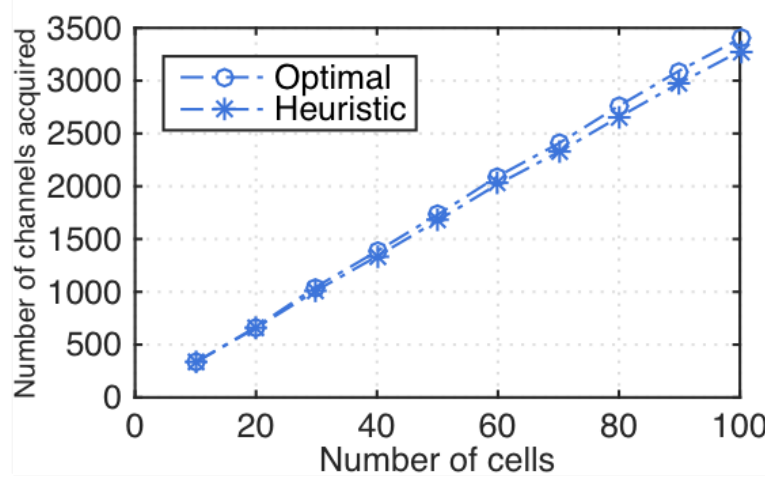

Fig. 7: Bandwidth acquisition of the SNO for spectrum borrowing by the optimal and heuristic algorithms.

We also study how the optimal allocation based on profit maximisation affects the amount of acquired bandwidths. With number of cells between 1 and 100, we compare the two algorithms presented to solve Problem 2, see Figure 7. We find that, the optimal algorithm can achieve higher number of aggregated channels due to the higher efficiency in spectrum borrowing under the restricted budget.

In the proposed algorithms, we added a functionality to allow the trading to be managed more effectively by assigning each cell with a particular band type. In order to quantify the impact of the proposed algorithms we simulated a network which could support three different bands, $(M=3)$. We also tested the algorithms with two different budgets. In the simulation of 10 cells 
and allocated budget of 50 and 500 for each cell, we observed a markedly increased profit in both cases, see Figure 8. We can also see from the figures (top and bottom figures) that in all types of bands, the optimal algorithm outperforms its heuristic counterpart.
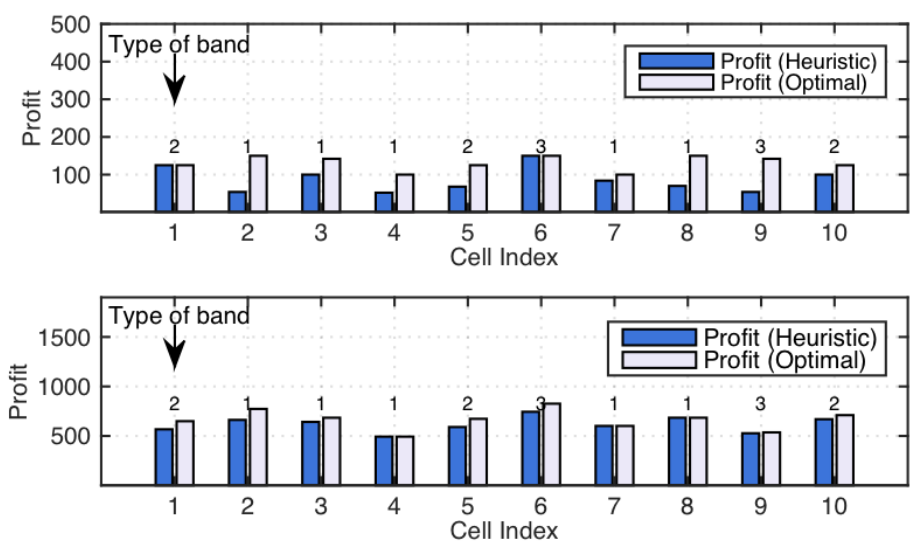

Fig. 8: Effect of spectrum borrowing on profit with budget $=50$ (top) and budget $=500$ (bottom).

\section{Post-optimization performance of the operators}

To analyze the impact of unilateral deviation strategy of the PNOs, we used the closed form formulae presented in Section III-G to compute the blocking probability of operators. The arrival processes involved in all operators are non-homogenous Poisson with rates $\lambda_{s_{1}}, \lambda_{s_{2}}, \lambda_{1}, \lambda_{2}$, and $\lambda_{3}$, respectively. The offered loads are $\lambda_{s} / \mu_{s}$ and $\lambda_{i} / \mu_{i}$ for the $s$ th secondary and $i$ th PNO, respectively. The number of aggregated channels and traffic intensities in each operator are independent as shown in table II. The results show that the operators could obtain an actual blocking probability values to determine their benefits when they engage in spectrum trading.

In Figure 9a, we observe the performance of the PNOs and the SNOs by varying the traffic load at the PNOs (from 1 to 5). If we fix a particular value of traffic intensity at the SNOs ( $\rho_{s_{1}}=1$ and $\rho_{s_{2}}=5$ ) and change it for the PNOs, then the SNOs' blocking probability slightly increase due to the available capacity for sharing $\left(c_{1}^{\prime}, c_{2}^{\prime}\right.$ and $\left.c_{3}^{\prime}\right)$ become overloaded by the PNOs' own traffic. We notice that the severity of traffic intensity change in the PNOs affects the performance of the SNO.

In Figure 9b, we analyse the impact of change in state of the PNOs from overloaded to underloaded. As the shared capacity becomes ample to meet the SNOs' demand, we notice a significant reduction in blocking probability at the SNOs. We also notice that the blocking 
TABLE II: Configurations used in Figure 9a and 9b.

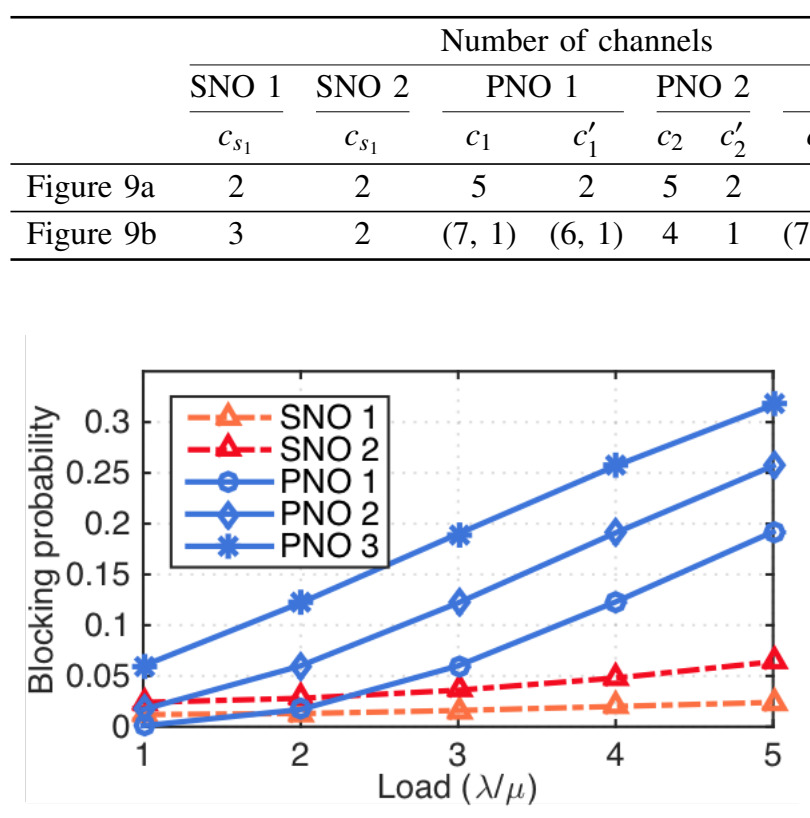

(a)

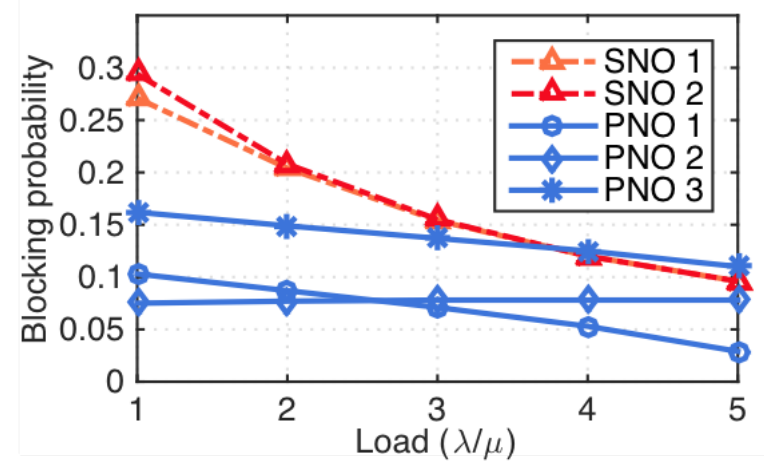

(b)

Fig. 9: Blocking probability for each operator when configuration details of (a) and (b) are according to Table II.

probability of PNO 2 is not affected by the changes in state of other primary and secondary operators since its shared capacity and traffic load remains constant for the duration. The results demonstrate that the derived blocking probabilities can provide a crucial insight to the sharing strategies between operators.

\section{CONCLuSion}

In this paper, we presented two finite horizon nonlinear optimization algorithms to solve two optimization problems for dynamic spectrum sharing. The efficiency of the proposed algorithms is compared with their corresponding heuristic algorithms. We also presented the post-optimization performance analysis of the SNO and PNOs through blocking probability, which provides useful details about spectrum sharing strategy.

The optimization problems investigated by considering a comprehensive process of delivering the SNO's bandwidth demand and the solution algorithms ensured that either minimum cost of bandwidth borrowing or maximum profit under budget restrictions are achieved depending on the aim of the SNO. In both cases the SNO aims to achieve a target performance by borrowing 
spectrum from PNOs on temporal and spatial basis. Detailed comparisons are presented and they showed that the gain in the results obtained from our proposed stochastic-optimization framework is markedly higher than heuristic borrowing algorithms. Our proposed approaches facilitate a dynamic purchasing (also called automation of licensing) scheme for such complex problems, which provide incentives to the network operators wishing to adopt dynamic spectrum sharing as well as substantial benefits for efficient use of spectrum. The proposed algorithms showed significant opportunities to increase spectrum utilisation while keeping GoS at a particular level and ensuring minimum cost. We also shown that our proposed optimization solution not only reduce the total borrowing cost of the SNOs, but also finds maximum spectrum access under any allocated budget.

A major challenge with the spectrum sharing optimization models is to guarantee the operational GoS under different sharing protocols. Although a vast amount of literature addressed various spectrum sharing issues very little has discussed the post-optimization results which are crucial for the operators to gain the detailed insight and final GoS. To study these issues and provide the final GoS, we derived the blocking probability behavior using a time-dependent continuous time Markov chain framework. Results showed that the final GoS is largely affected by the increase of traffic at the PNOs and the amount of shared resources. This post-optimization analysis of spectrum sharing among the operators is an emerging topic that requires further research that would cover other issues, for instance, different sharing strategies and configurations.

\section{REFERENCES}

[1] A. Palaios, J. Riihijarvi, P. Mahonen, V. Atanasovski, L. Gavrilovska, P. Van Wesemael, A. Dejonghe, and P. Scheele, "Two days of spectrum use in Europe," in 7th International Conference on Cognitive Radio Oriented Wireless Networks and Communications, 2012, pp. 24-29.

[2] V. Valenta, R. Maršálek, G. Baudoin, M. Villegas, M. Suarez, and F. Robert, "Survey on spectrum utilization in europe: Measurements, analyses and observations," in Fifth International Conference on Cognitive Radio Oriented Wireless Networks and Communications, 2010, pp. 1-5.

[3] A. A. Khan, M. H. Rehmani, and M. Reisslein, "Cognitive radio for smart grids: Survey of architectures, spectrum sensing mechanisms, and networking protocols," IEEE Communications Surveys \& Tutorials, vol. 18, no. 1, pp. 860-898, 2016.

[4] G. Ding, J. Wang, Q. Wu, Y.-D. Yao, F. Song, and T. A. Tsiftsis, "Cellular-base-station-assisted device-to-device communications in tv white space," IEEE J. Sel. Areas Commun., vol. 34, no. 1, pp. 107-121, 2016.

[5] T. S. Rappaport et al., Wireless communications: principles and practice. New Jersey: Prentice Hall, 1996. 
[6] H. Zhang, C. Jiang, X. Mao, and H.-H. Chen, "Interference-limited resource optimization in cognitive femtocells with fairness and imperfect spectrum sensing," IEEE Transactions on Vehicular Technology, vol. 65, no. 3, pp. 1761-1771, 2016.

[7] A. Afana, V. Asghari, A. Ghrayeb, and S. Affes, "On the performance of cooperative relaying spectrum-sharing systems with collaborative distributed beamforming," IEEE Transactions on Communications, vol. 62, no. 3, pp. 857-871, 2014.

[8] L. Wei, R. Q. Hu, Y. Qian, and G. Wu, "Energy efficiency and spectrum efficiency of multihop device-to-device communications underlaying cellular networks," IEEE Transactions on Vehicular Technology, vol. 65, no. 1, pp. 367$380,2016$.

[9] Y. Xiao, Z. Han, C. Yuen, and L. A. DaSilva, "Carrier aggregation between operators in next generation cellular networks: A stable roommate market," IEEE Transactions on Wireless Communications, vol. 15, no. 1, pp. 633-650, 2016.

[10] R. Abozariba, M. Asaduzzaman, and M. Patwary, "Radio resource sharing framework for cooperative multi-operator networks with dynamic overflow modelling," IEEE Transactions on Vehicular Technology, 2016, In press.

[11] A. Ghosh and S. Sarkar, "Quality-sensitive price competition in secondary market spectrum oligopoly- single location game," IEEE/ACM Transactions on Networking, vol. 24, no. 3, pp. 1894-1907, 2016.

[12] G. Kasbekar, S. Sarkar, K. Kar, P. Muthuswamy, and A. Gupta, "Dynamic contract trading in spectrum markets," IEEE Transactions on Automatic Control, vol. 59, no. 10, pp. 2856-2862, 2014.

[13] M. Asaduzzaman, R. Abozariba, and M. Patwary, "Spectrum sharing optimization in cellular networks under target performance and budget restriction,” in 85th IEEE Vehicular Technology Conference, 2017.

[14] A. Ullah, S. Bhattarai, J.-M. Park, J. H. Reed, D. Gurney, and B. Bahrak, "Multi-tier exclusion zones for dynamic spectrum sharing," in 2015 IEEE International Conference on Communications, 2015, pp. 7659-7664.

[15] Y. Zhang, C. Lee, D. Niyato, and P. Wang, "Auction approaches for resource allocation in wireless systems: A survey," IEEE Communications Surveys \& Tutorials, vol. 15, no. 3, pp. 1020-1041, 2013.

[16] C. A. Gizelis and D. D. Vergados, "A survey of pricing schemes in wireless networks," IEEE Communications Surveys \& Tutorials, vol. 13, no. 1, pp. 126-145, 2011.

[17] A.-H. Mohsenian-Rad, V. W. Wong, and V. Leung, "Two-fold pricing to guarantee individual profits and maximum social welfare in multi-hop wireless access networks," IEEE Transactions on Wireless Communications, vol. 8, no. 8, pp. 41104121, 2009.

[18] N. Tran, L. B. Le, S. Ren, Z. Han, and C. S. Hong, "Joint pricing and load balancing for cognitive spectrum access: Non-cooperation versus cooperation,” IEEE J. Sel. Areas Commun., vol. 33, no. 5, pp. 972-985, May 2015.

[19] S. Sengupta and M. Chatterjee, "An economic framework for dynamic spectrum access and service pricing," IEEE/ACM Transactions on Networking, vol. 17, no. 4, pp. 1200-1213, 2009.

[20] J. W. Mwangoka, P. Marques, and J. Rodriguez, "Broker based secondary spectrum trading," in Sixth International Conference on Cognitive Radio Oriented Wireless Networks and Communications, 2011, pp. 186-190.

[21] D. S. Palguna, D. J. Love, and I. Pollak, "Secondary spectrum auctions for markets with communication constraints," IEEE Transactions on Wireless Communications, vol. 15, no. 1, pp. 116-130, 2016.

[22] M. Khaledi and A. A. Abouzeid, "Dynamic spectrum sharing auction with time-evolving channel qualities," IEEE Transactions on Wireless Communications, vol. 14, no. 11, pp. 5900-5912, 2015.

[23] Y. Wu, Q. Zhu, J. Huang, and D. H. Tsang, "Revenue sharing based resource allocation for dynamic spectrum access networks," IEEE J. Sel. Areas Commun., vol. 32, no. 11, pp. 2280-2296, 2014. 
[24] S. Li, J. Huang, and S.-Y. R. Li, "Dynamic profit maximization of cognitive mobile virtual network operator," IEEE Transactions on Mobile Computing, vol. 13, no. 3, pp. 526-540, 2014.

[25] I. Sugathapala, I. Kovacevic, B. Lorenzo, S. Glisic, and Y. M. Fang, "Quantifying benefits in a business portfolio for multi-operator spectrum sharing," IEEE Transactions on Wireless Communications, vol. 14, no. 12, pp. 6635-6649, 2015.

[26] Y. Song, C. Zhang, Y. Fang, and P. Lin, "Revenue maximization in time-varying multi-hop wireless networks: A dynamic pricing approach,” IEEE J. Sel. Areas Commun., vol. 30, no. 7, pp. 1237-1245, 2012.

[27] L. Gao, J. Huang, Y.-J. Chen, and B. Shou, "An integrated contract and auction design for secondary spectrum trading," IEEE J. Sel. Areas Commun., vol. 31, no. 3, pp. 581-592, 2013.

[28] X. Zhu, L. Shen, and T.-S. Yum, "Analysis of cognitive radio spectrum access with optimal channel reservation," IEEE Communications Letters, vol. 11, no. 4, pp. 304-306, Apr. 2007.

[29] Q. Huang, Y.-C. Huang, K.-T. Ko, and V. Iversen, "Loss performance modeling for hierarchical heterogeneous wireless networks with speed-sensitive call admission control," IEEE Transactions on Vehicular Technology, vol. 60, no. 5, pp. 2209-2223, Jun. 2011.

[30] M. Matinmikko, H. Okkonen, M. Palola, S. Yrjola, P. Ahokangas, and M. Mustonen, "Spectrum sharing using licensed shared access: the concept and its workflow for LTE-advanced networks," IEEE Wireless Communications, vol. 21, no. 2, pp. 72-79, 2014.

[31] M. M. Buddhikot and K. Ryan, "Spectrum management in coordinated dynamic spectrum access based cellular networks," in First IEEE International Symposium on New Frontiers in Dynamic Spectrum Access Networks, 2005, pp. $299-307$.

[32] G. Li and H. Liu, "Downlink radio resource allocation for multi-cell ofdma system," IEEE Transactions on Wireless Communications, vol. 5, no. 12, pp. 3451-3459, 2006

[33] M. M. Buddhikot, P. Kolodzy, S. Miller, K. Ryan, and J. Evans, "DIMSUMnet: new directions in wireless networking using coordinated dynamic spectrum," in Sixth IEEE International Symposium on a World of Wireless Mobile and Multimedia Networks, 2005, pp. 78-85.

[34] K. I. Aardal, S. P. Van Hoesel, A. M. Koster, C. Mannino, and A. Sassano, "Models and solution techniques for frequency assignment problems," Quarterly Journal of the Belgian, French and Italian Operations Research Societies, vol. 1, no. 4, pp. 261-317, 2003. 